\title{
Original
}

\section{Encuesta sobre Condiciones de Seguridad y Salud en el Trabajo para Ecuador. Principales resultados en la ciudad de Quito, 2016}

\section{Survey on Safety and Health Conditions at the Workplace in Ecuador. Main Results in Quito, 2016}

\author{
Antonio R. Gómez García' ', Pamela Merino-Salazar², Michael Silva-Peñaherrera ${ }^{3}$, Pablo R. Suasnavas Bermúdez², \\ Aimee Vilaret Serpa ${ }^{2}$
}

1. Universidad Espíritu Santo, Samborondón, Ecuador.

2. Universidad Internacional SEK, Quito, Ecuador.

3. Universitat Pompeu Fabra, Barcelona, España.

Recibido: 12-11-2019

Aceptado: 16-12-2019

\section{Correspondencia}

Antonio Ramón Gómez García

Universidad de Especialidades Espíritu Santo

Samborondón, Guayaquil. Ecuador

agomezg@uees.edu.ec

Resumen

Objetivo: Describir las condiciones de trabajo y el estado de salud de la población trabajadora afiliada a la seguridad social de la ciudad de Quito, Ecuador.

Materiales y métodos: Estudio descriptivo basado en los resultados de la Primera Encuesta sobre Condiciones de Seguridad y Salud en el Trabajo (I-ECSST). Se entrevistó entre abril y junio de 2016 a 741 personas en su domicilio. El diseño muestral fue probabilístico, bietápico y estratificado por las 32 parroquias urbanas de la ciudad de Quito.

Resultados: Un porcentaje considerable de los trabajadores estaba expuesto a factores de riesgos ergonómicos. En particular, el $60 \%$ de los trabajadores encuestados manifestó realizar movimientos repetitivos en su actividad laboral. Se observó una alta prevalencia de dolores o molestias de espalda (50\%), cabeza (40\%) y miembros superiores (26\%). El 13\% de los trabajadores declaró haber sufrido un accidente de trabajo en el último año.

Conclusiones: La I-ECSST revela multitud de factores de riesgos laboral presentes en los lugares de trabajo que pueden estar ocasionando daños a la salud de la población trabajadora. Estos resultados podrían servir para guiar a las políticas públicas orientadas a mejorar la salud de la población trabajadora.

Med Segur Trab (Internet). 2019;65(257):238-251

Palabras clave: encuestas epidemiológicas, condiciones de trabajo, factores socioeconómicos, Ecuador.

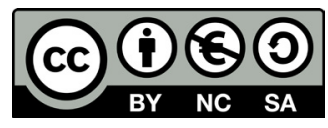

https://creativecommons.org/licenses/by-nc-sa/4.0/ 


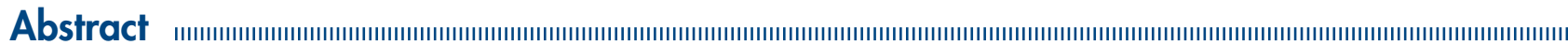

Objective: To describe working conditions and health problems at the working-age population in the Ecuadorian social security system of Quito.

Materials and methods: Descriptive study based on the results of the First Survey on Safety and Health at Work (I-ECSST, by its Spanish acronym). 741 people were interviewed at home between April and June 2016. The sampling design was probabilistic, multistage and stratified by 32 urban parishes.

Results: A considerable proportion of workers were exposed to ergonomic risk factors. In particular, $60 \%$ of surveyed workers evidenced repetitive movements throughout their work activity. A high prevalence of pains and problems were evidenced as follows: back (50\%), head (40\%) and upper limb (26\%). 13\% of workers reported having suffered a work-related accident in the previous year.

Conclusions: The I-ECSST reveals a multitude of occupational risk factors at workplaces that may be causing damage to the health of the working population. These results could be helpful in guiding public policies in order to improve the health of the working population.

Med Segur Trab (Internet). 2019;65(257):238-251

Keywords: Epidemiological surveys, working conditions, socioeconomic factors, Ecuador. 


\section{INTRODUCCIÓN}

Los factores de riesgo laboral presentes en los lugares de trabajo pueden tener una influencia significativa en la generación de daños a la salud de la población trabajadora. De la misma manera, producen pérdidas productivas para las empresas y tienen un impacto económico negativo que repercute principalmente en el crecimiento de los países de medianos y bajos ingresos ${ }^{1,2,3}$. Diferentes estudios consideran esta problemática, no sólo desde la perspectiva económica, sino también, como un tema prioritario en salud pública por los efectos negativos que suponen los accidentes de trabajo y enfermedades profesionales a la sociedad ${ }^{4}$.

No obstante, uno de los grandes obstáculos para el diseño de políticas públicas enfocadas a proteger la salud de la población trabajadora, especialmente en estos países, es la falta de información fiable. Consecuentemente, la I y II Estrategia Iberoamericana de Seguridad y Salud en el Trabajo manifiestan la necesidad de conocer la situación actual de las condiciones de trabajo y salud de los trabajadores en cada país, destacando a las encuestas sobre condiciones de trabajo y salud (ECTS) como el instrumento más recomendado para describir, analizar y realizar un seguimiento a lo largo del tiempo en este ámbito ${ }^{5,6}$.

Las ECTS se han consolidado como una herramienta eficaz para obtener información, tanto sobre las condiciones de empleo, como sobre los factores de riesgo presentes en los puestos de trabajo y su impacto en la salud de la población trabajadora ${ }^{7}$. De esta manera, su principal objetivo es guiar en el diseño y evaluación de programas nacionales y políticas públicas. Asimismo, constituyen una oportunidad para la investigación en salud laboral ${ }^{8,9}$.

Las ECTS se han venido implementando desde hace más de 40 años ${ }^{10}$, destacando la Encuesta Europea de Condiciones de Trabajo por sus altos parámetros de calidad y su amplia cobertura geográfica ${ }^{11}$. En América Latina y El Caribe estas encuestas tienen un desarrollo reciente, sin embargo, han proporcionado por primera vez, información válida y fiable para conocer y comparar las condiciones de trabajo y salud entre países ${ }^{12,13}$, tema prioritario de salud y desarrollo planteado por los organismos internacionales ${ }^{14,15,16}$. Sin embargo, en el contexto ecuatoriano no se han realizado estudios sobre esta materia.

El objetivo del presente estudio es dar a conocer, por primera vez, los resultados más relevantes obtenidos a través de la aplicación de la Primera Encuesta sobre Condiciones de Seguridad y Salud en el Trabajo (I-ECSST), desde la percepción de la población trabajadora en la capital del Ecuador ${ }^{17,18}$.

La información presentada permite describir el estado actual de la salud laboral en la población objeto de estudio, a través de la agrupación de variables socio-demográficas, laborales y factores de riesgo presentes en los lugares de trabajo, además, es útil como complemento a los datos estadísticos sobre siniestralidad laboral ${ }^{19,20}$.

\section{MATERIALES Y MÉTODOS}

Estudio descriptivo y transversal basado en los datos procedentes de la aplicación de la I-ECSST en la ciudad de Quito, Ecuador ${ }^{18}$.

La I-ECSST fue realizada en la capital del país entre los meses de abril y junio de 2016, a personas ocupadas de 18 años o mayores, con relación de dependencia, afiliados al seguro social y que declararon estar trabajando en el momento de realizar la entrevista. El muestreo fue probabilístico, bietápico y estratificado por las 32 parroquias urbanas de la ciudad (división político-territorial de menor rango circunscrita dentro de la ciudad).

En la primera etapa, dentro de cada estrato, se seleccionaron las viviendas mediante un muestreo sistemático con arranque aleatorio. El número de viviendas en cada estrato se determinó mediante afijación proporcional, tomando en cuenta la cantidad de 
moradores en el último censo poblacional disponible al momento de la realización de la encuesta (Censo de Población y Vivienda 2010 del Instituto Nacional de Estadística y Censos del Ecuador). En la segunda etapa, se seleccionó a la persona trabajadora dentro del domicilio por cuotas establecidas según sexo. Cuando hubo más de una persona que cumplía con los criterios establecidos, se seleccionó al entrevistado por muestreo aleatorio simple. La muestra final estuvo conformada por 741 personas (364 mujeres y 377 hombres).

Previamente, se realizó una prueba piloto (pre-test) a 56 personas (hombres y mujeres) en el mes de marzo de 2016 con el objetivo de verificar el grado de comprensión de las preguntas y respuestas del cuestionario.

El trabajo de campo se llevó a cabo través de una compañía encuestadora especializada. Previamente, el equipo de encuestadores fue capacitado por los investigadores en cuanto a los objetivos, formulación e interpretación de las preguntas, además, periódicamente se supervisó la aplicación de la encuesta para garantizar la calidad de los datos. El cuestionario, conformado por un total de 79 preguntas, se aplicó mediante entrevista personal en el domicilio de la persona trabajadora, con una duración entre 30 a 90 minutos.

El diseño del cuestionario de la I-ECCTS se basó en un modelo conceptual que toma en cuenta la compleja relación entre el trabajo remunerado y la salud ${ }^{17}$. Este modelo sitúa a las condiciones de trabajo, que incluye las condiciones de seguridad, ambiente físico, contaminantes químicos y biológicos, carga física de trabajo y factores de riesgo psicosocial, a nivel central. Además, también considera a las condiciones de empleo como determinantes fundamentales de la salud de la población trabajadora, específicamente, a las horas de trabajo remunerado y los turnos de trabajo. Asimismo, incorpora las características sociodemográficas (sexo, edad, nivel educativo y nacionalidad) y laborales (actividad económica, ocupación y tamaño de la empresa). Finalmente, el modelo incluye diversos daños a la salud, como, por ejemplo, accidentes laborales, enfermedades profesionales, autopercepción de la salud e incapacidades. Por lo tanto, a partir de las dimensiones de la salud de la población trabajadora y sus distintos determinantes, tomadas en cuenta en este modelo conceptual, el presente estudio presenta los resultados más relevantes de la I-ECCTS, desde el punto de vista descriptivo.

\section{RESULTADOS}

\section{Características socio-demográficas}

La Tabla 1 muestra las características socio-demográficas de la población trabajadora encuestada $(n=741)$. Con respecto a la distribución por edad, la mayor parte son trabajadores menores de 45 años, con una edad de 37,9 $\pm 10,1$ años, predominando los ecuatorianos $(98,7 \%)$ respecto a los de otra nacionalidad $(1,3 \%)$.

En la distribución por niveles educativos, no se observaron diferencias significativas por sexo. Sin embargo, hay un mayor porcentaje de hombres en relación con las mujeres que han alcanzado estudios de bachillerato. Por el contrario, una menor proporción de hombres frente a las mujeres han obtenido el cuarto nivel de posgrado.

\section{Características laborales y empleo}

Los trabajadores encuestados se concentran mayormente en el sector privado respecto al público. En cuanto al puesto de trabajo que ocupan, el 22,4\% (n=166) corresponde a puestos de servicios o vendedores, el 20,4\% ( $n=151)$ administrativos, el $18,5 \%(n=137)$ actividades de apoyo y técnicos y/o profesionales de nivel medio el 17,0\% $(n=126)$. En función al tamaño de empresa, se evidencia que un $25 \%$ de las mujeres y un $20 \%$ de los hombres trabajan en grandes empresas en su mayoría del sector de servicios (Tabla 2). 
La modalidad más extendida es la jornada completa (8 horas diarias) en horario diurno. Además, un 20\% manifiesta trabajar más de 40 horas semanales (23,9\% en hombres y $15,1 \%$ en mujeres).

Tabla 1. Características socio-demográficas según sexo de la población trabajadora afiliada a la seguridad social en la ciudad de Quito $n=741$ (\%).

\begin{tabular}{|c|c|c|c|c|c|c|}
\hline & \multicolumn{2}{|r|}{ Mujeres } & \multicolumn{2}{|r|}{ Hombres } & \multicolumn{2}{|r|}{ Total } \\
\hline & $n=364$ & $\%(I C 95 \%)$ & $\mathrm{n}=\mathbf{3 7 7}$ & $\%($ IC95\%) & $n=741$ & $\%(I C 95 \%)$ \\
\hline \multicolumn{7}{|l|}{ Edad } \\
\hline 18 a 24 & 26 & $7,1(4,5-9,8)$ & 25 & $6,6(4,1-9,1)$ & 51 & $6,9(5,1-8,7)$ \\
\hline 25 a 34 & 142 & $39(34,0-44,0)$ & 121 & $32,1(27,4-36,8)$ & 263 & $35,5(32-38,9)$ \\
\hline $35 \mathrm{a} 44$ & 110 & $30,2(25,5-34,9)$ & 114 & $30,2(25,6-34,9)$ & 224 & $30,2(26,9-33,5)$ \\
\hline 45 a 55 & 66 & $18,1(14,2-22,1)$ & 86 & $22,8(18,6-27,0)$ & 152 & $20,5(17,6-23,4)$ \\
\hline 56 a 64 & 18 & $4,9(2,7-7,2)$ & 27 & $7,2(4,6-9,8)$ & 45 & $6,1(4,4-7,8)$ \\
\hline$\geq 65$ & 2 & $0,5(0-1,3)$ & 4 & $1,1(0-2,1)$ & 6 & $0,8(0,2-1,5)$ \\
\hline \multicolumn{7}{|l|}{ Nacionalidad } \\
\hline Ecuatoriana & 358 & $98,4(97-99,7)$ & 373 & $98,9(97,9-100)$ & 731 & $98,7(97,8-99,5)$ \\
\hline Otra & 6 & $1,6(0,3-3)$ & 4 & $1,1(0-2,1)$ & 10 & $1,3(0,5-2,2)$ \\
\hline \multicolumn{7}{|l|}{ Nivel Educativo } \\
\hline Menos de Básica & 2 & $0,5(0,-1,3)$ & 2 & $0,5(0-1,3)$ & 4 & $0,5(0-1,1)$ \\
\hline Educación general básica & 18 & $4,9(2,7-7,2)$ & 16 & $4,2(2,2-6,3)$ & 34 & $4,6(3,1-6,1)$ \\
\hline Educación bachillerato & 118 & $32,4(27,6-37,2)$ & 143 & $37,9(33,0-42,8)$ & 261 & $35,2(31,8-38,7)$ \\
\hline Tecnológico superior & 88 & $24,2(19,8-28,6)$ & 83 & $22(17,8-26,2)$ & 171 & $23,1(20,0-26,1)$ \\
\hline Tercer nivel de grado & 107 & $29,4(24,7-34,1)$ & 114 & $30,2(25,6-34,9)$ & 221 & $29,8(26,5-33,1)$ \\
\hline Cuarto nivel de postgrado & 31 & $8,5(5,6-11,4)$ & 19 & $5(2,8-7,2)$ & 50 & $6,7(4,9-8,6)$ \\
\hline
\end{tabular}

* Ubicación geográfica del domicilio del trabajador encuestado de la ciudad de Quito.

Tabla 2. Distribución por actividad económica y tamaño de empresa de la población trabajadora afiliada a la seguridad social de la ciudad de Quito $n=741$ (\%).

\begin{tabular}{|c|c|c|c|c|c|c|c|c|c|c|}
\hline & \multicolumn{8}{|c|}{ Tamaño de Empresa } & \multirow{2}{*}{\multicolumn{2}{|c|}{ Total }} \\
\hline & \multicolumn{2}{|r|}{$\leq 9$} & \multicolumn{2}{|r|}{10 a 49} & \multicolumn{2}{|r|}{50 a 199} & \multicolumn{2}{|r|}{$\geq 200$} & & \\
\hline & \multirow{2}{*}{$\mathbf{n}$} & $\%$ & \multirow{2}{*}{$\mathbf{n}$} & $\%$ & \multirow{2}{*}{$\mathbf{n}$} & $\%$ & \multirow{2}{*}{ n } & $\%$ & \multirow{2}{*}{$\mathrm{n}$} & $\%$ \\
\hline & & (IC95\%) & & (IC95\%) & & (IC95\%) & & (IC95\%) & & (IC95\%) \\
\hline \multicolumn{11}{|l|}{ Actividad Económica } \\
\hline \multirow{2}{*}{ Agricultura } & \multirow{2}{*}{1} & 0,1 & \multirow{2}{*}{2} & 0,3 & \multirow{2}{*}{-} & \multirow{2}{*}{-} & \multirow{2}{*}{3} & 0,4 & \multirow{2}{*}{6} & 0,8 \\
\hline & & $(0-0,4)$ & & $(0-0,6)$ & & & & $(-0,1-0,9)$ & & $(0,2-1,5)$ \\
\hline \multirow{2}{*}{ Comercio } & \multirow{2}{*}{23} & 3,1 & \multirow{2}{*}{27} & 3,6 & \multirow{2}{*}{18} & 2,4 & \multirow{2}{*}{16} & 2,2 & \multirow{2}{*}{84} & 11,3 \\
\hline & & $(1,9-4,4)$ & & $(2,3-5)$ & & $(1,3-3,5)$ & & $(1,1-3,2)$ & & $(9,1-13,6)$ \\
\hline \multirow{2}{*}{ Construcción } & \multirow{2}{*}{6} & 0,8 & \multirow{2}{*}{9} & 1,2 & \multirow{2}{*}{8} & 1,1 & \multirow{2}{*}{3} & 0,4 & \multirow{2}{*}{26} & 3,5 \\
\hline & & $(0,2-1,5)$ & & $(0,4-2)$ & & $(0,3-1,8)$ & & $(-0,1-0,9)$ & & $(2,2-4,8)$ \\
\hline \multirow{2}{*}{$\begin{array}{l}\text { Explotación minas y } \\
\text { canteras }\end{array}$} & \multirow{2}{*}{-} & \multirow{2}{*}{ - } & 2 & 0,3 & & & 3 & 0,4 & 5 & 0,7 \\
\hline & & & 2 & $(0-0,6)$ & & - & $J$ & $(-0,1-0,9)$ & J & $(0,1-1,3)$ \\
\hline Ind untrios m fo & 7 & 0,9 & 16 & 2,2 & 10 & 2,4 & & 2,6 & 60 & 8,1 \\
\hline mutstras mamuractureras & 1 & $(0,2-1,6)$ & 10 & $(1,1-3,2)$ & 10 & $(1,3-3,5)$ & 19 & $(1,4-3,7)$ & 00 & $(6,1-10,1)$ \\
\hline & & 12,3 & & 22,8 & & 15,9 & & 24,6 & & 75,6 \\
\hline Servicios & 91 & $(9,9-14,6)$ & 169 & $(19,8-25,8)$ & 118 & $(13,3-18,6)$ & 182 & $(21,5-27,7)$ & 560 & $\begin{array}{l}(72,5- \\
78,7)\end{array}$ \\
\hline & 128 & 17,3 & 225 & 30,4 & 162 & 21,9 & 226 & 30,5 & & \\
\hline Iotal & 128 & $(14,6-20)$ & $2 Z 5$ & $\overline{(27,1-33,7)}$ & 102 & $\overline{(18,9-24,8)}$ & 220 & $\overline{(27,2-33,8)}$ & 14 & 100 \\
\hline
\end{tabular}




\section{Condiciones de seguridad}

Los hombres se encuentran en mayor medida expuestos a riesgos de accidentes de trabajo (riesgo mecánico) respecto a las mujeres. Los riesgos que con mayor frecuencia declaran los trabajadores encuestados son los cortes - pinchazos - rozaduras $(24,8 \%)$, golpes $(22,8 \%)$, caída de objetos, materiales o herramientas $(17,9 \%)$ y proyección de partículas (15,9\%). La exposición a estos factores de riesgo es más habitual entre los hombres en actividades económicas de la construcción e industria, sin observar diferencias en cuanto al tamaño de la empresa, Tabla 3.

Otros riesgos de menor frecuencia de exposición, pero igualmente considerables, son la caída a distinto nivel, incendios, contactos eléctricos (directos - indirectos) y accidentes de tránsito. Es importante resaltar que el 10,9\% de los trabajadores manifestaron riesgo de sufrir un accidente de tránsito.

Tabla 3. Exposición a riesgo mecánico según sexo de la población trabajadora afiliada a la seguridad social de la ciudad de Quito (\%).

\begin{tabular}{lcccc}
\hline & \multicolumn{2}{c}{ Mujeres } & \multicolumn{2}{c}{ Hombres } \\
\cline { 2 - 5 } & $\mathbf{n}$ & $\%(\mathbf{I C 9 5} \%)$ & $\mathbf{n}$ & $\%(\mathbf{I C 9 5} \%)$ \\
\hline Riesgo de caída al mismo nivel & 39 & $10,7(7,5-13,9)$ & 62 & $16,4(12,7-20,2)$ \\
\hline Riesgo de caída a distinto nivel & 39 & $10,7(7,5-13,9)$ & 60 & $15,9(12,2-19,6)$ \\
\hline Riesgo de caída de objetos, materiales... & 54 & $14,8(11,2-18,5)$ & 79 & $21(16,8-25,1)$ \\
\hline Riesgo de desplomes-derrumbamientos & 44 & $12,1(8,7-15,4)$ & 56 & $14,9(11,3-18,4)$ \\
\hline Riesgo de cortes, pinchazos... & 85 & $23,4(19-27,7)$ & 99 & $26,3(21,8-30,7)$ \\
\hline Riesgo de golpes & 72 & $19,8(15,7-23,9)$ & 97 & $25,7(21,3-30,1)$ \\
\hline Riesgo de atropellos por vehículos & 21 & $5,8(3,4-8,2)$ & 47 & $12,5(9,1-15,8)$ \\
\hline Riesgo de atrapamiento con equipos... & 23 & $6,3(3,8-8,8)$ & 48 & $12,7(9,4-16,1)$ \\
\hline Riesgo de proyección de partículas... & 53 & $14,6(10,9-18,2)$ & 65 & $17,2(13,4-21,1)$ \\
\hline Riesgo a quemaduras & 51 & $14(10,4-17,6)$ & 59 & $15,6(12-19,3)$ \\
\hline Daños exposición al sol & 18 & $4,9(2,7-7,2)$ & 56 & $14,9(11,3-18,4)$ \\
\hline Riesgo de incendios-explosiones & 40 & $11(7,8-14,2)$ & 54 & $14,3(10,8-17,9)$ \\
\hline Daños por animales & 11 & $3(1,3-4,8)$ & 12 & $3,2(1,4-5)$ \\
\hline Riesgo a contactos eléctricos & 43 & $11,8(8,5-15,1)$ & 57 & $15,1(11,5-18,7)$ \\
\hline Riesgo de accidentes de tránsito & 25 & $6,9(4,3-9,5)$ & 56 & $14,9(11,3-18,4)$ \\
\hline
\end{tabular}

\section{Equipos de protección personal}

El 37\% de los trabajadores señalan que durante su trabajo están obligados a utilizar equipos de protección individual, destaca el calzado de seguridad (25\%), guantes para riesgo mecánico (21\%), mascarilla facial (18\%) y casco de seguridad (17\%), principalmente empleado por los hombres del sector construcción e industrial.

\section{Ambiente Físico de Trabajo}

Respecto al ambiente físico en los lugares de trabajo, se presentan los resultados de aquellos trabajadores que manifestaron estar expuestos siempre o casi siempre en su jornada laboral.

El 24\% de los hombres y el 16\% de las mujeres respondieron estar expuestos a ruido (confort acústico) durante su jornada laboral, impidiéndoles mantener una conversación con otra persona que este a tres metros de distancia.

Uno de cada cuatro hombres manifiesta estar expuesto a vibraciones en miembros superiores (mano - brazo), cuyo origen procede del uso de vehículos de transporte (33\%), herramientas portátiles (24\%) y maquinaria (23\%). 
El $70 \%$ percibe la temperatura (confort térmico) como idónea en su puesto de trabajo, no obstante, un $9 \%$ indica que la humedad es demasiada seca y el $6 \%$ excesivamente húmeda. Todos los porcentajes para los agentes físicos son mayores en hombres que en mujeres.

\section{Contaminantes químicos y biológicos}

Uno de cada cinco trabajadores manipula sustancias químicas (siempre o casi siempre) en su actividad laboral, un $18 \%$ de los hombres inhala alguno de los productos químicos en forma de polvos, humos, gases o vapores que considera nocivos o tóxicos para la salud.

El $86 \%$ de los trabajadores refiere que los productos químicos que manipulan disponen de etiqueta informativa. Por último, las mujeres (11\%) presentan mayor exposición por contacto a microorganismos, virus y bacterias que los hombres (6\%) siempre o casi siempre durante su jornada laboral.

\section{Carga física de trabajo}

Más del $60 \%$ de los trabajadores realizan movimientos repetitivos durante su jornada laboral, sin presentarse diferencias significativas por sexo. El 15\% manipulan, levantan o trasladan cargas u otros objetos pesados siempre o casi siempre en sus tareas $(20,4 \%$ en hombres; $10,2 \%$ en mujeres).

Las posturas habituales de trabajo son la de sentado (33\% levantándose con frecuencia y sin levantarse casi nunca un 20\%) y de pie caminado frecuentemente (41\%). Aproximadamente un tercio realizan trabajos que obligan a mantener posturas incómodas (siempre el 7,4\%, casi siempre el 6,2\% y el 16,5\% algunas veces), Figura 1.

Figura 1. Distribución porcentual de la carga física de trabajo de la población trabajadora afiliada a la seguridad social de la ciudad de Quito (\%).

Sentado $(n=392)$

De pié $(n=339)$

Posturas Incomodas $(\mathrm{n}=101)$

Manipulación manual de cargas ( $n=114)$

Movimientos repetitivos $(n=456)$

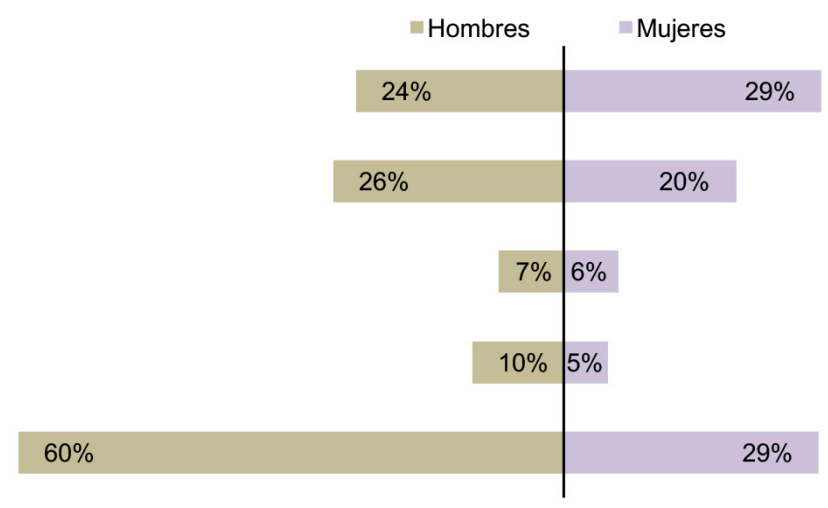

\section{Factores de riesgo psicosocial}

En cuanto a los resultados obtenidos en los factores de riesgo psicosociales, destaca un $33 \%$ de los trabajadores que declaran un trato negativo por parte de los compañeros, siendo las mujeres $(65,6 \%)$ el grupo que mayor sufre este tipo de conductas y amenazas violentas en su lugar de trabajo.

La totalidad de trabajadores encuestados consideran poseer los conocimientos, habilidades y tiempo suficiente para realizar las tareas exige su puesto de trabajo. El 61\% manifiesta realizar esfuerzos mentales para cumplir con su trabajo, sin observar diferencias significativas por sexo. Mientras que la mayoría afirma sentirse apoyado por los compañeros de trabajo, solo un $7 \%$ de hombres y $9 \%$ de mujeres no perciben el apoyo de sus jefes o superiores. 
Se evidenció un alto porcentaje de trabajadores que respondieron sentir temor de perder su trabajo actual (67\% en hombres y $64 \%$ en mujeres).

\section{Estilo de Vida}

Uno de cada tres encuestados no respeta los horarios establecidos para la alimentación. El 74\% considera que su trabajo le permite dormir el número de horas necesarias para recuperarse y un porcentaje mayoritario ( $95 \%$ de hombres y $94 \%$ de mujeres) declara realizar alguna actividad física, recreativa, cultural u otra en su tiempo libre.

Un hecho a destacar es que, el $26 \%$ de los trabajadores declara consumir alguna sustancia como el tabaco, alcohol y/o café debido a las preocupaciones relacionadas con su trabajo, en particular hombres con edades entre los 18 a 34 años y con estudios universitarios.

Considerando la conciliación con la vida familiar y su relación con el trabajo, el 38\% de los trabajadores percibe que su trabajo actual interfiere en el tiempo dedicado a la familia y el 43\% está preocupado y tensionado por el tiempo invertido en el traslado del domicilio al lugar de trabajo y viceversa.

\section{Estado de salud de la población trabajadora}

El 91\% de los trabajadores considera su estado de salud como bueno, muy bueno o excelente. Por el contrario, un $37 \%$ percibe que su trabajo actual puede afectar negativamente a su salud, principalmente en mujeres con edades comprendidas entre los 25 a 55 años.

Referente a la salud mental, el 18\% declara haber padecido alguna depresión o algún tipo de problema emocional durante el último mes.

Entre los principales problemas de salud destaca la alta prevalencia de dolores o molestias de espalda (50\%), cabeza (40\%) y miembros superiores (26\%), predominando más en mujeres hasta los 40 años de edad. También son frecuentes, por orden de importancia, los problemas oftalmológicos, respiratorios, digestivos y auditivos.

En la Tabla 4 se presenta la distribución porcentual de los problemas de salud percibidos por los trabajadores encuestados ajustados según sexo y edad.

En cuanto al análisis por actividad económica, los trabajadores del sector industrial y servicios son los que presentan mayores problemas de salud, en particular en espalda (50\%) y miembros superiores.

El 13\% de los trabajadores declara haber sufrido un accidente de trabajo en el último año, (8.4\% son hombres y 4.6\% mujeres), siendo el grupo más afectado los trabajadores entre los 25 a 55 años de edad, con más de 9 años de antigüedad en la empresa y de menor nivel educativo.

La agricultura es la actividad económica proporcionalmente con mayor accidentabilidad (50\%), seguido de la industria (27\%) y construcción (19\%).

El 51\% de los casos de accidentes originó una incapacidad temporal impidiéndole acudir al trabajo uno o más días. Destaca el sector servicios como la actividad con mayor reincidencia de accidentes de trabajo e incapacidad temporal.

Finalmente, el $8 \%$ manifiesta haber sufrido una enfermedad relacionada con el trabajo, de este porcentaje, el $63 \%$ ha sido reconocida legalmente por el Seguro General de Riesgos del Trabajo del Instituto Ecuatoriano de Seguridad Social, un 12\% se encuentra en trámite de calificación y no ha sido reconocida en un $25 \%$ de los casos. 
Tabla 4. Problemas de salud según sexo y edad de la población trabajadora afiliada a la seguridad social de la ciudad de Quito (\%).

\begin{tabular}{|c|c|c|c|c|c|c|}
\hline \multirow[b]{3}{*}{ Mujeres } & \multicolumn{6}{|c|}{ Rango de Edad } \\
\hline & \multicolumn{2}{|r|}{$18-34$} & \multicolumn{2}{|r|}{$35-55$} & \multicolumn{2}{|r|}{$>55$} \\
\hline & $n=168$ & \%(IC95\%) & $\mathrm{n}=176$ & \%(IC95\%) & $\mathrm{n}=\mathbf{2 0}$ & $\%(I C 95 \%)$ \\
\hline \multicolumn{7}{|l|}{ Molestias o Dolor } \\
\hline Cabeza & 78 & $46,4(38,9-54)$ & 80 & $45,5(38,1-52,8)$ & 6 & $30,0(9,9-50,1)$ \\
\hline Espalda & 96 & $57,1(49,7-64,6)$ & 96 & $54,5(47,2-61,9)$ & 12 & $60,0(38,5-81,5)$ \\
\hline Miembros Superiores & 46 & $27,4(20,6-34,1)$ & 55 & $31,3(24,4-38,1)$ & 9 & $45,0(23,2-66,8)$ \\
\hline \multicolumn{7}{|l|}{ Problemas } \\
\hline Auditivos & 21 & $12,5(7,5-17,5)$ & 14 & $8,0(4,0-12,0)$ & 5 & $25,0(6,0-44,0)$ \\
\hline Cardiocirculatorios & 4 & $2,4(0,1-4,7)$ & 11 & $6,3(2,7-9,8)$ & 4 & $20,0(2,5-37,5)$ \\
\hline Dermatológicos & 15 & $8,9(4,6-13,2)$ & 15 & $8,5(4,4-12,6)$ & 4 & $20,0(2,5-37,5)$ \\
\hline Digestivos & 22 & $13,1(8,0-18,2)$ & 30 & $17,0(11,5-22,6)$ & 6 & $30,0(9,9-50,1)$ \\
\hline Oftalmológicos & 32 & $19(13,1-25)$ & 35 & $19,9(14-25,8)$ & 8 & $40,0(18,5-61,5)$ \\
\hline Respiratorios & 25 & $14,9(9,5-20,3)$ & 28 & $15,9(10,5-21,3)$ & 2 & $10,0(0-23,1)$ \\
\hline Hombres & $n=146$ & \%(IC95\%) & $\mathrm{n}=\mathbf{2 0 0}$ & \%(IC95\%) & $\mathrm{n}=31$ & $\%(I C 95 \%)$ \\
\hline \multicolumn{7}{|l|}{ Molestias o Dolor } \\
\hline Cabeza & 49 & $33,6(25,9-41,2)$ & 76 & $38,0(31,3-44,7)$ & 11 & $35,5(18,6-52,3)$ \\
\hline Espalda & 67 & $45,9(37,8-54)$ & 86 & $43,0(36,1-49,9)$ & 11 & $35,5(18,6-52,3)$ \\
\hline Miembros Superiores & 34 & $23,3(16,4-30,1)$ & 40 & $20,0(14,5-25,5)$ & 10 & $32,3(15,8-48,7)$ \\
\hline \multicolumn{7}{|l|}{ Problemas } \\
\hline Auditivos & 16 & $11,0(5,9-16)$ & 29 & $14,5(9,6-19,4)$ & 9 & $29,0(13,1-45,0)$ \\
\hline Cardiocirculatorios & 4 & $2,7(0,1-5,4)$ & 10 & $5,0(2,0-8,0)$ & 1 & $3,2(0-9,4)$ \\
\hline Dermatológicos & 14 & $9,6(4,8-14,4)$ & 16 & $8,0(4,2-11,8)$ & 3 & $9,7(0-20,1)$ \\
\hline Digestivos & 27 & $18,5(12,2-24,8)$ & 24 & $12,0(7,5-16,5)$ & 5 & $16,1(3,2-29,1)$ \\
\hline Oftalmológicos & 26 & $17,8(11,6-24)$ & 40 & $20,0(14,5-25,5)$ & 6 & $19,4(5,4-33,3)$ \\
\hline Respiratorios & 19 & $13(7,6-18,5)$ & 39 & $19,5(14-25)$ & 5 & $16,1(3,2-29,1)$ \\
\hline
\end{tabular}

\section{DISCUSIÓN}

Este es el primer estudio que analiza las condiciones de trabajo y salud utilizando una muestra representativa de la población mayor a 18 años, con relación de dependencia, afiliados al Seguro Social y con contrato laboral vigente en la capital del Ecuador.

Del análisis de los resultados cabe señalar unas condiciones de trabajo y salud muy similares al resto de países de Latinoamérica y El Caribe ${ }^{12,13}$. La muestra que representa a la población trabajadora de Quito, se caracteriza por ser joven, (edad media de 37,9 $\pm 10,1$ años), a diferencia de los países desarrollados dónde existe un mayor porcentaje de trabajadores en edad avanzada ${ }^{21}$. Dado que para las próximas décadas se prevé un envejecimiento de la población trabajadora ecuatoriana, la edad constituye una variable básica de análisis, puesto que condiciona las capacidades tanto físicas como mentales y puede generar con mayor probabilidad patologías de origen laboral, como se ha visto en varios estudios que confirman la relación existente entre la edad y estado de salud ${ }^{22}$.

Con relación a las condiciones de empleo, cabe destacar que una alta proporción de la población trabaja más de 40 horas semanales, especialmente los hombres. La variable tiempo de trabajo ha sido considerada de suma importancia para la I-ECSST, ya que la cantidad de horas que destinamos al trabajo remunerado puede ser considerada como determinante en el aumento de la probabilidad de sufrir accidentes de trabajo, aparición 
de enfermedades profesionales e interfiere en la conciliación de la vida familiar del trabajador ${ }^{23,24}$.

En relación a los riesgos de accidentes percibidos, se constata la existencia de riesgos tradicionales como los cortes - pinchazos - rozadura, golpes, caída de objetos, materiales o herramientas y proyección de partículas. El 37\% de los trabajadores señalan que durante su trabajo están obligados a utilizar equipos de protección individual, datos similares a los hallados en la encuesta de España $(41 \%)^{25}$. Del mismo modo, encontramos para los riesgos físicos que uno de cada cinco trabajadores manipula sustancias químicas en su actividad laboral, un $18 \%$ de los hombres inhala alguno de los productos químicos en forma de polvos, humos, gases o vapores que considera nocivos o tóxicos para la salud.

Por último, las mujeres presentan mayor exposición a agentes biológicos. Este resultado puede ser debido, en parte, a una mayor proporción de mujeres en actividades sanitarias o una mayor conciencia de estas sobre los riesgos biológicos ${ }^{26,27}$.

Siguiendo un patrón similar de exposición entre la población estudiada y otros países que han empleado como fuente de información las encuestas de condiciones de trabajo y salud, los factores de riesgo ergonómico están entre las exposiciones más frecuente. Por ejemplo, según la VI Encuesta Europea de Condiciones de Trabajo el 69\% de la población trabajadora en 35 países ${ }^{21}$ y $61 \%$ en España ${ }^{25}$ están expuestos a movimientos repetitivos. Como indicamos anteriormente, estos factores de riesgo pueden incrementar la probabilidad de que se produzcan daños a la salud, originando a corto y/o medio plazo la aparición de trastornos musculo - esqueléticos de origen laboral en la población estudiada $^{28}$.

Con respecto a los factores de riesgo psicosocial, un porcentaje importante de trabajadores señala que ha de enfrentarse a sentimientos, emociones o trato negativo de otras personas y no perciben el apoyo de sus jefes o superiores. En este sentido, la gestión de los recursos humanos en las empresas debe incorporar acciones encaminadas al estudio de situaciones de violencia, acoso o discriminación sufridas por el trabajador, principalmente en el grupo de mujeres. Además, cabe destacar la preocupación de los trabajadores por ser despedidos, la inseguridad en el empleo en estos momentos de crisis económica puede generar, no sólo precariedad en el empleo debido a los contratos temporales, sino también, condiciones inseguras en los lugares de trabajo que puedan impactar de forma negativa en la salud de los trabajadores, debido principalmente a la reducción de los recursos económicos y actividades preventivas en las empresas ${ }^{29,30}$. Estudios recientes encuentran una correlación negativa entre siniestralidad laboral y crisis económica, fenómeno observado en las pequeñas y medianas empresas de países en desarrollo ${ }^{2,31}$.

En cuanto a la salud auto percibida, el 91\% de los trabajadores considera su estado de salud como bueno, muy bueno o excelente, porcentaje inferior a Colombia, pero notablemente superior a los porcentajes encontrados en Centroamérica y Chile $\mathrm{e}^{12,13}$. Este hallazgo llama la atención, ya que a pesar de que la salud depende de muchos determinantes, de alguna manera, podemos considerar que la población trabajadora está conforme con su trabajo actual y calidad de vida, a pesar de que un $37 \%$ perciben que su trabajo actual puede afectar negativamente a su salud.

Destaca la alta prevalencia de dolor de espalda, cabeza y miembros superiores, predominando más en mujeres hasta los 40 años ${ }^{13,28}$. Estos resultados coinciden con lo observado a través de la I Encuesta Centroamericana de Condiciones de Trabajo y Salud, donde más del $50 \%$ de la población trabajadora en cuatro países refería dolor musculoesquelético ${ }^{32}$. También son frecuentes, por orden de importancia, los problemas oftalmológicos, respiratorios, digestivos y auditivos.

El 13\% de los trabajadores declara haber sufrido un accidente de trabajo en el último año, principalmente los hombres con edades comprendidas entre 25 a 55 años de edad y con menor nivel educativo, esta situación podría estar relacionada con el acceso de los trabajadores de menor educación a puestos de trabajo menos calificados y dónde las 
actividades diarias que desarrollan entrañan mayor nivel de riesgo laboral. Estos hallazgos, coindicen con las estadísticas oficiales y estudios similares en el país ${ }^{20}$. De los 20.296 accidentes de trabajo calificados a nivel nacional en 2016 , el 78,9\% fueron hombres entre los 26 a 55 años de edad (75,4\%), fenómeno que igualmente coincide con la siniestralidad laboral de Colombia ${ }^{33}$ y Perú $^{34}$. Es importante señalar que un $51 \%$ de los casos de accidentes causaron ausentismo laboral de más de un día. La incapacidad temporal permite ser un indicador importante para determinar la gravedad de los accidentes de trabajo y evidencian condiciones inseguras en los lugares de trabajo analizados ${ }^{35}$.

Este estudio no está exento de limitaciones similares e inherentes a otras encuestas internacionales ${ }^{13}$. En primer lugar, si bien la percepción de los trabajadores es considerada como una desventaja, esta información es un elemento central para conocer las condiciones de seguridad y salud en los lugares de trabajo. Además, ciertas preguntas como la salud auto percibida, que se basa esencialmente en la percepción del trabajador sobre su propia salud, ha demostrado ser una buena predictora de morbilidad $^{9,36}$. En segundo lugar, al tratarse de una muestra representativa de la población trabajadora ocupada de 18 años o más, con relación de dependencia y afiliada al Seguro Social de la ciudad de Quito, estos hallazgos no se pueden generalizar a nivel nacional y representar a la población desempleada o con empleo informal. Los últimos datos disponibles del Censo de Población y Vivienda del 2010, muestran que únicamente el 27.6\% (32.3\% de los hombres y 23.7 de las mujeres) de la población ocupada con relación de dependencia y residente en las parroquias urbanas de Quito estaban afiliadas al seguro social ${ }^{37}$. Datos más recientes de la Encuesta Nacional de Empleo, Desempleo y Subempleo (ENEMDU), muestran que el 29.1\% de la población ocupada con relación de dependencia del país estaba afiliada al seguro social a marzo del $2017^{38}$. Esto sugiere que aproximadamente el $70 \%$ de la población ocupada de Quito no ha sido considerada en el presente estudio. Finalmente, durante el trabajo de campo se presentaron incidencias: desconfianza y resistencia del trabajador entrevistado a la hora de proporcionar datos acerca de sus condiciones de trabajo, seguridad y estado de salud; para la obtención de 1 encuesta efectiva bajo los criterios de inclusión fue necesario abordar aproximadamente a 7 personas y; los encuestadores encontraron un predominio de hombres según los criterios de inclusión.

Pese a estas limitaciones, los resultados presentados en este estudio permiten conocer, por primera vez, las condiciones de trabajo y salud de la población trabajadora afiliada en la ciudad de Quito. En futuras investigaciones debería estudiarse las condiciones de trabajo y salud en el resto de provincias del país para realizar estudios comparativos, ya que existen diferencias socioculturales y económicas. Asimismo, el disponer de nuevas versiones de la encuesta permitiría evaluar las tendencias temporales en el estado de salud de la población trabajadora. En conclusión, sería muy recomendable que la encuesta incluyera a la población con empleo informal ya que representa un porcentaje significativo de la población trabajadora y ha sido vinculada con pobres condiciones de empleo (por ejemplo: empleo temporal y largas jornadas de trabajo) y trabajo, y por lo tanto con daños a la salud ${ }^{39,40}$. Todo esto facilitaría la toma de decisiones basadas en una mejor evidencia.

Están programados futuros análisis más específicos, en particular, la comparación de los resultados de la I-ECSST con las encuestas realizadas en países en Latinoamérica y El Caribe $^{12,13}$, así como, estudios sobre desigualdades de género en las condiciones de empleo, trabajo, conciliación de la vida laboral y familiar, y problemas de salud.

En resumen, los resultados de esta encuesta para la ciudad Quito revelan multitud de factores de riesgos laboral presentes en los lugares de trabajo, éstos pueden originar daños a la salud en la población trabajadora para los próximos años. Finalmente, debemos puntualizar que la información analizada puede ser complementaria a las estadísticas sobre siniestralidad laboral ${ }^{19,20}$ y línea base para la formulación de nuevas políticas y la propuesta de un plan nacional en seguridad y salud en el trabajo para el Ecuador.

Consideraciones éticas: el trabajo de campo de la aplicación I-ECSST se realizó garantizando el anonimato y confidencialidad de las trabajadoras y trabajadores encuestados 


\section{REFERENCIAS BIBLIOGRÁFICAS}

1. De la Fuente VS, López MA, González IF, Alcántara OJ, Ritzel DO. The Impact of the economic crisis on occupational injuries. Journal of Safety Research. 2014; 48:77-85. doi: 10.1016/j.jsr.2013.12.007

2. Mrema EJ, Ngowi AV, Mamuya SHD. Status of occupational health and safety and related challenges in expanding economy of Tanzania. Annals of Global Health. 2015; 81(4):538-547. doi: 10.1016/j. aogh.2015.08.021

3. Colak $\mathrm{O}$, Palaz S. The relationship between economic development and fatal occupational accidents: Evidence from Turkey. Scientific Annals of Economics and Business. 2017; 64(1):19-31. doi: 10.1515/saeb2017-0002

4. Riaño Casallas MI, Palencia Sánchez F. Los costos de la enfermedad laboral: revisión de literatura. Revista Facultad Nacional de Salud Pública. 2015; 33(2):218-227. doi: 10.17533/udea.rfnsp.v33n2a09

5. Organización Iberoamericana de Seguridad Social. I Estrategia Iberoamericana de Seguridad y Salud en el Trabajo 2010-2013 [Internet]. OISS; 2010 [citado el 2 de septiembre de 2017]. Disponible en: http:// www.oiss.org/IMG/pdf/EISST_mayo2010_.pdf

6. Organización Iberoamericana de Seguridad Social. II Estrategia Iberoamericana de Seguridad y Salud en el Trabajo 2015-2020 [Internet]. OISS; 2013 [citado el 2 de septiembre de 2017]. Disponible en: http:// www.srt.gob.ar/images\%5Cpdf\%5CEISST-2015-2020.pdf

7. Formazin M, Burr H, Aagestad C, Tynes T, Thorsen SV, Perkio Makela M,...Houtman I. Dimensional comparability of psychosocial working conditions as covered in European monitoring questionnaires. BMC Public Health. 2014; 9(14):1251. doi: 10.1186/1471-2458-14-1251

8. Itatí Iñiguez MJ, Agudelo Suárez AA, Campos Serna J, Cornelio C, Benavides F. Encuestas de condiciones de trabajo y salud: su utilización en la investigación en salud laboral. Medicina y Seguridad del Trabajo. 2012; 58(228):205-215.

9. Caro V, Alba Y, Agudelo S, Andrés A, Benavides F. Relación entre las condiciones de trabajo y el estado de salud en la población trabajadora afiliada al Sistema General de Riesgos Profesionales de Colombia. Revista Facultad Nacional de Salud Pública. 2011; 29(4):392-401i. doi: 10.1186/1471-2458-14-1251

10. Merino Salazar P, Artazcoz L, Campos Serna J, Gimeno D, Benavides FG. National working conditions surveys in Latin America: comparison of methodological characteristics. International Journal of Occupational and Environmental Health. 2015; 21(3):266-274. doi: 10.1179/2049396715Y.0000000004

11. Cabrita J, Peycheva D. National working conditions surveys in Europe: A compilation. Luxembourg: European Foundation for the Improvement of Living and Working Conditions [Internet]. Eurofound; 2014 [citado el 26 de septiembre de 2017]. Disponible en: http://digitalcommons.ilr.cornell.edu/cgi/viewcontent. cgi?article $=1403 \&$ context $=$ intl

12. Merino Salazar P, Artazcoz L, Cornelio C, Itatí Iñiguez MJ, Rojas M, Martínez Iñigo D, Vives A, Funcasta L, Benavides F. Work and health in Latin America: results from the working conditions surveys of Colombia, Argentina, Chile, Central America and Uruguay. Occupational and Environmental Medicine. 2017; 74(6):432-439. doi: 10.1136/oemed-2016-103899

13. Benavides F, Wesseling C, Delclos G, Felknor S, Pinilla J, Rodrigo F. Working conditions and health in Central America: A survey of 12,024 workers in six countries. Occupational and Environmental Medicine. 2014; 71(7):459-465. doi: 10.1136/oemed-2013-101908

14. World Health Organization. Workers' Health: Global Plan of Action 2008-2017 [Internet]. WHO; 2007. [citado el 12 de octubre de 2017]. Disponible en: http://www.who.int/occupational_health/WHO_health_ assembly_en_web.pdf.

15. Organización Internacional del Trabajo. Programa de referencia de la OIT: Programa de Acción Global para la Prevención en el ámbito de la seguridad y salud en el trabajo - OSH-GAP [Internet]. OIT; 2015. [citado el 17 de octubre de 2017]. Disponible en: http://www.ilo.org/wcmsp5/groups/public/---ed_ dialogue/---lab_admin/documents/genericdocument/wcms_589067.pdf

16. Organización Internacional del Trabajo. Plan de implementación de la OIT Agenda 2030 para el Desarrollo Sostenible [Internet]. OIT; 2015. [citado el 19 de octubre de 2017]. Disponible en: http://www.ilo.org/ wcmsp5/groups/public/---dgreports/---dcomm/-- webdev/documents/publication/wcms_510124.pdf

17. Peñaherrera Silva MG, Gómez García AR, Vilaret Serpa A, Suasnavas Bermúdez PR. Diseño Metodológico de la Encuesta sobre Condiciones de Seguridad y Salud en el Trabajo en Ecuador. Innova Research Journal. 2016; 1(8):1-14.

18. Gómez García AR. I Encuesta sobre Seguridad y Salud en el Trabajo para Ecuador: I-ESST. CienciAmérica. 2017; 6(1):67-75.

19. Gómez García AR, Suasnavas Bermúdez PR. Incidencia de Accidentes de Trabajo Declarados en Ecuador en el Período 2011-2012. Revista Ciencia \& Trabajo. 2015; 52(5):49-53. doi: 10.4067/S0718-24492015000100010 
20. Gómez García AR, Algora Buenafé AF, Suasnavas Bermúdez PR, Silva Peñaherrera MG, Vilaret Serpa A. Notificación de Accidentes de Trabajo y Posibles Enfermedades Profesionales en Ecuador, 2010-2015. Revista Ciencia \& Trabajo. 2016; 18(57):166-172. doi: 10.4067/S0718-24492016000300166

21. European Foundation for the Improvement of Living and Working Conditions. Primeros resultados sexta Encuesta europea sobre las condiciones de trabajo [Internet]. Luxembourg: Publications Office; 2015 [citado el 28 de octubre de 2017]. Disponible en: http://bookshop.europa.eu/uri?target=EUB:NOTICE:TJ0 115746:ES:HTML

22. Pinilla García FJ, Almodóvar Molina A, Hervás Rivero P, Galiana Blanco L, Marta Zimmermann Verdejo M. La edad de las condiciones de trabajo. Resultados de la $6^{\text {a }}$ EWCS-España. Revista Seguridad y Salud en el Trabajo. 2016; 88:15-28.

23. Christian Moretti A. Duración de la jornada laboral: implicaciones sanitarias y político-económicas Revista Chilena de Terapia Ocupacional. 2015; 15(1):57-64

24. European Foundation for the Improvement of Living and Working Conditions Reconciliation of work and family life and collective bargaining in the European Union [Internet]. Eurofound; 2006. [citado el $30 \mathrm{de}$ octubre de 2017]. Disponible en: http://www.pedz.uni-mannheim.de/daten/edz-ma/esl/06/ef0606en.pdf

25. Instituto Nacional de Seguridad e Higiene en el Trabajo. Encuesta Nacional de Condiciones de Trabajo. $20156^{a}$ EWCS - España [Internet]. INSHT; 2017. [citado el 7 de septiembre de 2017]. Disponible en: http:// www.insht.es/InshtWeb/Contenidos/Documentacion/FICHAS\%20DE\%20PUBLICACIONES/EN\%20 CATALOGO/GENERALIDAD/ENCT\%202015.pdf

26. Boscarino JA, Adams RE. Assessing Community Reactions to Ebola Virus Disease and Other Disasters: Using Social Psychological Research to Enhance Public Health and Disaster Communications. International Journal of Emergency Mental Health. 2015; 17(1):234-238. doi: 10.4172/1522-4821.1000147

27. García López V. III Encuesta Navarra de Salud y Condiciones de Trabajo: Principales hallazgos. Archivos de Prevención de Riesgos Laborales. 2017; 20(2):102-110.

28. Merino Salazar P., Gómez García AR, Silva Peñaherrera GM, Suasnavas Bermúdez PR, Rojas M. (2018) The Impact of Ergonomic Exposures on the Occurrence of Back Pain or Discomfort: Results from the First Working Conditions Survey in Quito-Ecuador. In: Goossens R. (eds.) Advances in Social \& Occupational Ergonomics. AHFE 2017. Advances in Intelligent Systems and Computing, vol. 605. Springer, Cham. doi: 10.1007/978-3-319-60828-0_23

29. Benavides FG, Benach J, Muntaner C, Delclos GL, Catot N, Amable M. Association between temporary employment and occupational injury: what are the mechanisms? Occupational and Environmental Medicine. 2006; 63:416-421. doi: 10.1136/oem.2005.022301

30. Benavides FG. Crisis económica y salud laboral. Archivos de Prevención de Riesgos Laborales. 2009; 12(3):113-114.

31. Unnikrishnan S, Iqbal R, Singh A, Nimkar IM. Safety management practices in small and medium enterprises in India. Safety and Health at Work. 2015; 6(1):46-55. doi: 10.1016/j.shaw.2014.10.006

32. Rojas M, Gimeno D, Vargas Prada S, Benavides FG. Dolor musculoesquelético en trabajadores de América Central: resultados de la I Encuesta Centroamericana de Condiciones de Trabajo y Salud. Revista Panamericana de Salud Pública. 2015; 38(2):120-128

33. Pérez MM, Muñoz AM. Caracterización de la accidentalidad laboral reportada por las empresas afiladas a una Administradora de Riesgos Laborales ARL, enero - diciembre de 2011. Revista Facultad Nacional de Salud Pública. 2014; 32(2): 67-75

34. Mejía CR, Cárdenas MM, Gomero Cuadra R. Notificación de accidentes y enfermedades laborales al Ministerio de Trabajo. Perú 2010-2014. Revista Peruana de Medicina Experimental y Salud Pública. 2015; 32(3):526-31

35. Villaplana García M, Sáez Navarro C, Meseguer de Pedro M, García Izquierdo M. Grado de efecto de las variables sociodemográficas, laborales, organizativas y del entorno en la duración de la incapacidad temporal por contingencias comunes en España. Atención Primaria. 2015; 47(2):90-98. doi: 10.1016/j. aprim.2014.03.010

36. Benach J, Julià M, Tarafa G, Mir J, Molinero E, Vives A. La precariedad laboral medida de forma multidimensional: distribución social y asociación con la salud en Cataluña. Gaceta Sanitaria. 2015; 29(5):375-378. doi: 10.1016/j.gaceta.2015.04.002

37. Instituto Nacional de Estadística y Censos. Tabulados censales. Población de 12 y más años por aportación o afiliación al seguro, según provincia, cantón y parroquia de empadronamiento y sexo- 2010 [Internet]. INEC; 2010. [citado el 14 de febrero de 2019]. Disponible en: www.ecuadorencifras.gob.ec/informacioncensal-cantonal/

38. Instituto Nacional de Estadística y Censos. Reporte de Economía Laboral - marzo 2017 [Internet]. INEC 2017. [citado el 14 de febrero de 2019]. Disponible en: http://www.ecuadorencifras.gob.ec/documentos/ web-inec/EMPLEO/2017/Marzo/032017_Presentacion_M.Laboral.pdf 
39. Muntaner C, Solar O, Vanroelen C, Martínez J M, Vergara M, Santana V, Castedo A, Kim IlHo, Benach J. Unemployment, informal work, precarious employment, child labor, slavery, and health inequalities: pathways and mechanisms. International Journal of Health Services. 2010; 40(2):281-295. doi: 10.2190/ HS.40.2.h

40. López Ruiz M, Artazcoz L, Martínez JM, Benavides F. Informal employment and health status in Central America. BMC Public Health. 2015; 15:698. doi: 10.1186/s12889-015-2030-9

||||||||||||||||||||||||||||||||||||||||||||||||||||||||||||||||||||||||||||||||||||||||||||||||||||||||||||||||||||||||||||||||||||||||||||||||||||||||||||||||||||||||||||||||||||||||||

Contribuciones de los autores: Antonio Ramón Gómez García, Pamela MerinoSalazar y Michael Silva-Peñaherrera han participado en la concepción y diseño del artículo; análisis e interpretación de datos y redacción del artículo;

Pablo Roberto Suasnavas Bermúdez y Aimee Vilaret Serpa han participado en la revisión crítica del artículo y aprobación de la versión final.

Fuente de financiamiento: estudio financiado por la Universidad Internacional SEK - Ecuador, dentro del Programa sobre Condiciones de Seguridad y Salud en el Trabajo (Código P021516) de la Facultad de Ciencias del Trabajo y del Comportamiento Humano.

Conflictos de intereses: ninguno. 